\title{
L'utilisation des prises de notes dans le cadre d'une activité scientifique chez les enfants de 5 à 12 ans
}

La utilización de la toma de notas en el marco de una actividad científica en los niños de 5 a 12 años

Note-taking in the Context of a Scientific Activity among Children from 5 to 12 Years Old

A utilização de anotações no contexto de uma atividade científica em crianças de 5 a 12 anos

Date de réception: 20 FÉVRIER 2013/Date d'acceptation: 9 NOVEMBRE 2015/Date de disponibilité en ligne: 15 MARS 2016

Trouvez cet article dans: http://magisinvestigacioneducacion.javeriana.edu.co/

\section{Résumé}

La recherche qualitative présentée dans cet article a comme objectif d'étudier les prises de notes chez des enfants de 5 à 12 ans en tant que moyen de communication avec autrui. La situation expérimentale, comprenait une activité relative aux notions de "flottaison/immersion" des objets. Les enfants devaient, après l'avoir travaillé eux-mêmes, prendre des notes sur une feuille afin qu'un autre enfant puisse travailler dans le cadre de la même activité. Les résultats obtenus semblent supporter l'idée que les enfants de l'échantillon répondent à la demande de prendre des notes avec succès et avec une nette tendance à utiliser, suivant l'âge, l'écriture, ce qui montrerait l'influence de l'activité en tant que situation scolaire. On observe aussi une augmentation presque linéaire du degré d'exhaustivité et du degré de fonctionnalité des notations par rapport à l'âge des enfants.

\section{Mots-clés}

Expression écrite; enseignement des sciences; école primaire

\section{Transfert à la pratique}

Les résultats de cette recherche contribueront à éclaircir l'utilisation des prises de notes des enfants tant au niveau de mémorisation des situations expérimentales qu'à celui de la communication avec l'autre. Les enfants qui utilisent le dessin pour noter représentent un petit nombre de la population et ce qui paraît intéressant est la manière dont se différencient la fonctionnalité et l'exhaustivité des informations données par les enfants, relativement à la meilleure exploitation de l'activité.
Écrit par SPYRIDON TANTAROS UniveRsité D'ATHĖNES AtHènes, GrËCE sgtan@psych.uoa.gr

Konstantinos Ravanis Université de Patras Patras, Grèce ravanis@upatras.gr

Dimitris Koliopoulos Université de PATRAS Patras, Grèce dkoliop@upatras.gr

Kalypso Sarigianni Université de Patras Patras, Grèce sarigianni@upatras.gr

EVMORFIA SOTIROPOULOU Université de Patras PATRAs, Grèce esotiro@upatras.gr 
Palabras claves

Expresión escrita; enseñanza de las ciencias; escuela primaria

\section{Resumen}

La investigación cualitativa presentada en este artículo tiene como objetivo estudiar la toma de notas en los niños de 5 a 12 años como un medio de comunicación con el otro. La situación experimental, consistía en una actividad relativa a las nociones de flotación/inmersión de los objetos. Los niños debían, después de haber trabajado la actividad ellos mismos, tomar notas sobre una hoja con el objetivo de que otro niño pudiera trabajar en el marco de esta misma actividad. Los resultados obtenidos parecían sustentar la idea que los niños de la prueba responden a la petición de tomar notas con éxito y con una clara tendencia a utilizar, según la edad, la escritura, lo que muestra la influencia de la actividad como situación escolar. Se observó también un aumento de los apuntes de acuerdo a la edad de los niños.
Transferencia a la práctica

Los resultados de esta investigación contribuyeron a esclarecer la toma de notas de los niños tanto a nivel de memorización de las situaciones experimentales como al de la comunicación con el otro. Los niños que utilizan el dibujo para anotar representan un pequeño número de la población y lo que parece interesante es la manera en la que se diferencian la funcionalidad y la exhaustividad de las informaciones dadas por los niños, relativamente en el mejor desarrollo de la actividad.

\section{Keywords}

Written expression; science education; elementary school

\begin{abstract}
This paper, based on qualitative research, aims to study children note taking as a means to communicate with the other. The experimental situation was based on an activity regarding the notions of floating/ immersion of objects. After working in the activity, children needed to take notes on a paper so another peer could work within the framework of the same activity. Results seem to support the idea that children (from the test) respond to the request of taking notes successfully, and with a clear tendency, according to their age, to write. Thus, this research shows the influence of the activity. A note taking increase was also observed, according to children's age.
\end{abstract}

\section{Transfer to practice}

Results of this research contributed to clarifying children's abilities to take notes, both at the level of memorization in experimental situations and at communicating with the other. Children using drawings to write down their notes represent a small number of the population. It is interesting the way in which both functionality and exhaustiveness of the information provided by children differed during the activity.

\section{Palavras chave}

Expressão escrita; ensino das ciências; escola primária

\section{Resumo}

A pesquisa qualitativa apresentada neste artigo tem como objetivo estudar as anotações das crianças de 5 a 12 anos como um meio de comunicação com o outro. A situação experimental, consistia em uma atividade relacionada às noções de flutuação/ imersão de objetos. Após ter trabalhado a atividade, as mesmas crianças deveriam tomar notas sobre uma folha com o objetivo de que a outra criança pudesse trabalhar com esta mesma atividade. Os resultados obtidos pareciam sustentar a ideia que as crianças da amostra respondem à petição de tomar notas com sucesso e com uma clara tendência a utilizar, segundo a idade, a escrita, o que mostra a influência da atividade como situação escolar. Observou-se também um aumento dos apontamentos de acordo à idade das crianças.

\section{Transferência à prática}

Os resultados desta pesquisa ajudaram a esclarecer as anotações das crianças tanto a nível de memorização das situações experimentais como de comunicação com o outro. As crianças que utilizam o desenho para anotar representam um pequeno número da população e o que aparece como interessante é a maneira em que se diferenciam a funcionalidade e a exaustividade das informações dadas pelas crianças, relativamente ao melhor desenvolvimento da atividade. 


\section{Problématique théorique}

Dans le processus éducatif, I'apprentissage des codes symboliques occupe une place dominante. Cet apprentissage rend possible l'acquisition de toute connaissance future. Les codes les plus importants, constituant des connaissances fondamentales, sont enseignés à l'école: ce sont l'écriture et les symboles mathématiques. Contrairement à ce qui se passe pour les codes oraux analogues (Martí, 1997), ce sont des connaissances qui "s'apprennent" et qui donc doivent être enseignées.

Cependant, avant toute systématisation de la connaissance de ces codes, l'enfant est confronté à un certain nombre de stimuli qui retiennent I'attention des adultes, qui y répondent à leur tour par des comportements particuliers (par exemple, leur lecture ou des activités d'interprétation). II est presque certain que, de même qu'avec l'écriture (Ferreiro \& GómezPalacio, 1988), les enfants construisent des idées personnelles, font des hypothèses à propos de ces objets et essaient de les conceptualiser. Tout effort pour analyser cette connaissance "préalable" à l'enseignement de l'écriture poursuit un double objectif: comprendre la manière dont l'enfant approche l'enseignement de ces systèmes d'une part et définir, si possible, la nature de ce qui va être enseigné d'autre part (Garcia-Milà, Teberosky \& Martí, 2000; Martí \& Pozo, 2000).

La question de la didactique des sciences expérimentales, porte sur quand et comment le savoir doit être utilisé en relation aux autres activités didactiques... Les objectifs de départ étaient donc de chercher à mieux connaître le rôle des écrits dans les apprentissages scientifiques, en particulier dans l'appropriation des concepts scientifiques, de cerner quels types de textes, quels genres d'écrits favoriseraient ces apprentissages, de trouver des solutions didactiques aux difficultés des élèves (Catel, 2001). Certains auteurs font l'hypothèse "que d'une part, mieux écrire permet de mieux construire ses connaissances et que d'autre part, expérimenter et vivre une démarche d'investigation permettent de mieux écrire ce qui a été vécu" (Cros \& Respaud, 2001). D'autres se basent sur l'affirmation selon laquelle "les différentes formes d'écrits et les propriétés des langages eux-mêmes jouent un rôle dans la structuration de la pensée et la conceptualisation" (Fillon \& Vérin, 2001).

Les études sur l'écriture ont montré que la prise de notes constitue un cas particulier en ce qu'elle consiste en une procédure dont les chercheurs ont pointé la difficulté (Bovair \& Kieras, 1991; Ganier, 2002; Ganier, Gombert \& Fayol, 1998), en particulier lorsqu'il s'agit de décrire le fonctionnement des appareils techniques. Cette difficulté peut être expliquée de différentes manières: par la reconnaissance des relations entre l'action (savoir comment faire fonctionner un appareil) et la représentation (les notations), par la différenciation des fonctions de l'écriture et du dessin, etc.

Par ailleurs, les enfants rencontrent des difficultés analogues chaque fois qu'on leur demande de créer des notations relatives à des procédures durant lesquelles des objets et des substances entrent en contact et interagissent, contribuant ainsi à l'évolution des phénomènes physiques (Ravanis, 2011). En effet, la pensée des enfants est confrontée à une double difficulté qui du reste influence la méthodologie des recherches relatives à cette question. En leur demandant de produire des notations sur un phénomène physique, on les amène en même temps à produire les preuves de leur compréhension dudit phénomène, c'est-à-dire de leur possibilité de donner du sens à son évolution. Du point de vue éducatif, I'idéal serait qu'ils puissent donner un sens qui soit compatible avec celui des sciences physiques telles qu'on les approche dans l'enseignement
Description de l'article | Descripción del artículo | Article description | Artigo descrição

Cet article de la recherche présentée dans ce document examine les produits de notation des enfants de 5 à 12 ans comme un moyen de communication avec une autre personne. L'expérience a été basée sur un jeu lié à des objets "flottants" et "coulants". Après avoir joué à ce jeu, les enfants devaient écrire leurs propres instructions pour une autre personne qui voudrait jouer. Les résultats semblent soutenir que les enfants réussissent dans cette tâche, montrant une préférence pour l'écriture, en raison de l'influence de l'école. 
du même phénomène. Cette question est très importante dans le cadre de la didactique des sciences et c'est pourquoi une grande partie des recherches se tourne vers l'étude des représentations du monde naturel chez les enfants (Dumas-Carré, Weil-Barais, Ravanis \& Shourcheh, 2003; Fragkiadaki \& Ravanis, 2015). En révélant le rôle décisif des modèles de reconnaissance du monde naturel (tels qu'ils se construisent dans l'esprit des enfants avant l'enseignement) dans l'apprentissage, cette orientation de la recherche a complètement transformé les conceptions concernant le développement des processus didactiques et des activités provenant des sciences physiques. Une série de recherches a été réalisée dans cette perspective. Elles avaient comme objectif d'étudier les représentations mentales des enfants d'une part, d'essayer de transformer ces dernières afin qu'elles deviennent compatibles avec certains aspects descriptifs et fonctionnels des modèles scientifiques d'autre part (Ravanis, Christidou \& Hatzinikita, 2013; Ravanis, Papamichaël \& Koulaidis, 2002).

C'est dans ce cadre que se situe la recherche qui suit. Sur la base d'une situation expérimentale destinée à étudier la construction des notions de "submersion" et de "flottaison" des objets (Kohn, 1993; Weil-Barais \& Bouda, 2004), nous avons mené une première étude sur la manière dont les enfants de 5 à 12 ans essaient de noter des éléments de la procédure expérimentale "pour se rappeler" et "pour pouvoir dire après à leur camarade ce qu'ils ont fait".

Au niveau de l'éducation préscolaire et primaire, le phénomène de flottaison/submersion des objets solides tient une place centrale au sein des activités de routine concernant l'eau. Le plus souvent, les activités liées à la flottaison se limitent à faire classer des objets différents selon qu'ils coulent ou qu'ils flottent. Ces activités pourraient conduire à la construction de représentations formées au travers d'un processus d'abstraction empirique (Lemeignan \& Weil-Barais, 1993), telles que les concepts de "flottaison" et de "submersion", mais elles ne pourraient pas conduire à des suppositions sur la raison pour laquelle certains objets flottent et d'autres pas.

Les notions de "submersion" et de "flottaison" des objets est un champ de recherche déjà exploré dans des approches psycho-didactiques. Une première interprétation du phénomène de flottaison/submersion pourrait s'appuyer sur deux cadres conceptuels. Le premier est lié à la comparaison des valeurs des forces de poids et de la poussée d'Archimède qui s'exercent sur un objet. Le second est lié à la comparaison des densités de l'objet d'une part et du liquide dans lequel il se trouve de l'autre. Le premier cadre est basé sur une notion abstraite de la mécanique newtonienne du point de vue matériel, alors que le second suppose la définition du concept de "densité".

Se pose alors la question de savoir si les enfants de 5 à 12 ans parviennent à construire des modèles du phénomène de la flottaison/submersion des objets solides. Jean Piaget (1930/1974) a repéré quatre phases dans les explications que donnent les enfants de la flottaison des bateaux. Durant la première (qui finit vers l'âge de 5 ans), la flottaison est expliquée par des arguments de nature morale et animiste. Durant la seconde phase (de 5 à 6 ans), les enfants croient que les bateaux flottent parce qu'ils sont lourds, alors que de 6 à 8 ans ils sont persuadés du contraire. Durant la quatrième phase, vers 9 ans, ils commencent à comprendre la relation entre le poids des objets et la poussée d'Archimède.

Dans d'autres études, Jean Piaget et Bärbel Inhelder (1942/1974) aboutissent à la même conclusion pour le concept de densité. Ces résultats ont été confirmés par des recherches récentes qui, de plus, ont montré que les enfants identifient des facteurs tels que les cavités, les trous et la forme comme responsables de la flottaison des objets (Pramling \& 
Pramling-Samuelsson, 2001). Le résultat le plus attendu ici consisterait à montrer que dès l'âge préscolaire, les enfants sont susceptibles de former un modèle précurseur de densité (ou poids spécifique) sans faire référence au modèle analogue de "densité"/"masse"/"volume" (ou "poids spécifique"/"poids"/"volume"). Amy S. Kohn (1993) a trouvé que ces enfants ont une conception de la densité leur permettant de faire des prédictions exactes sur la poussée d'Archimède. Ce concept intuitif de la densité, que les enfants associent directement au matériau de l'objet, pourrait être le concept de base d'un modèle précurseur, descriptif du phénomène de flottaison/submersion, qui soit à la fois scientifiquement valable et adapté aux possibilités cognitives des enfants de cet âge.

Dans une expérience antérieure visant à explorer la possibilité qu'ont les petits enfants de construire un tel modèle précurseur pour la flottaison/ submersion des objets, a été demandé de prédire la flottaison/submersion possible de cubes de matériaux différents et de tailles différentes. Une fois leurs pronostics exprimés, les chercheurs leur a demandé de comparer leurs prédictions avec les résultats de l'expérience et d'en donner l'explication. Leurs réponses indiquent qu'un certain nombre d'entre eux avaient en fait construit leur propre modèle précurseur basé sur la nature du matériel, phénomène qu'accompagnait une amélioration générale de la qualité de leur raisonnement (Koliopoulos, Tantaros, Papandreou \& Ravanis, 2004).

L'objectif de la recherche de Sabrina Patricia Canedo-Ibarra, Josep Castelló-Escandell, Paloma García-Wehrle et Alejandro Rafael MoralesBlake (2010) a été d'explorer une expérimentation basée sur un dialogue avec des enfants de 6 ans pour la construction d'un modèle de flottaison basé sur la densité. L'étude exploratoire a été basée sur une analyse qualitative en utilisant un dessin dans chaque phase de la recherche (préenseignement, intervention didactique, et post-enseignement). Analysant les réponses des enfants après la phase d'instruction les chercheurs ont constaté que plusieurs enfants ont pu construire le modèle et ont montré en général un haut niveau qualitatif de raisonnement.

Ainsi, assurés qu'au niveau cognitif le phénomène de flottaison/submersion avait déjà un sens pour les enfants de notre échantillon, nous avons voulu, dans la recherche présentée ici, étudier l'évolution de l'activité de prise de note des enfants de 5 à 12 ans, tant au niveau de la mémorisation des situations expérimentales qu'à celui de la communication avec I'autre (Dixon, Faries \& Gabrys, 1998). Pour ce faire, nous avons demandé aux enfants de prendre des notes en vue de se rappeler quels sont les objets qui flottent et quels sont ceux qui coulent, afin d'être capables "d'aider leurs camarades à jouer au même jeu avec succès".

\section{Méthodologie}

\section{Échantillon et recueil des données}

Cette recherche qualitative est une "étude de cas" (Yin, 2014). L'échantillon se composait de 91 élèves, partagés en trois groupes d'âge (groupe A: 28 enfants de 5-6 ans, groupe B: 31 enfants de 7-8 ans et groupe C: 32 enfants de 11-12 ans), provenant de la maternelle (âge 5-6) et de la deuxième (âge 7-8) et sixième classe (âge 11-12) d'une école primaire privée d'Athènes. Les sujets de notre échantillon, n'ont pas reçu auparavant d'intervention didactique organisée dans l'école sur les notions de "flottaison/immersion" des objets. L'échantillon de l'enquête inclus des enfants de 5-6 ans, parce qu'à la fin de l'école maternelle en Grèce, ils commencent à être familiarisés avec l'écriture et à produire des écrits simples. Cette option est également partie de la nature évolutive du projet de recherche. 
Le choix des enfants a été fait uniquement sur la base de leur volonté de collaboration à "un jeu". On a introduit cette activité comme un "jeu" étant donné que ce choix construit un cadre communicationnel qui permet aux enfants s'exprimer d'une façon fonctionnelle et active.

La recherche, qui a duré deux semaines, a été réalisée par le biais d'entretiens individuels dans une partie de l'école qui se trouvait loin de la classe. La durée de chaque entretien était de 10 à 20 minutes environ. Tous les entretiens ont été filmés et analysés. La catégorisation du matériel a été faite par deux chercheurs indépendants, dont les choix ont été vérifiés par la suite. Les choix finaux ont été faits avec l'accord des cinq chercheurs qui y ont participé.

\section{Dispositifs et matériel expérimentaux}

Le matériel consistait en une maquette adaptée aux deux histoires. Celles-ci réclamaient un lac et ses alentours (Photo 1).

\section{Photo 1}

La maquette de l'activité

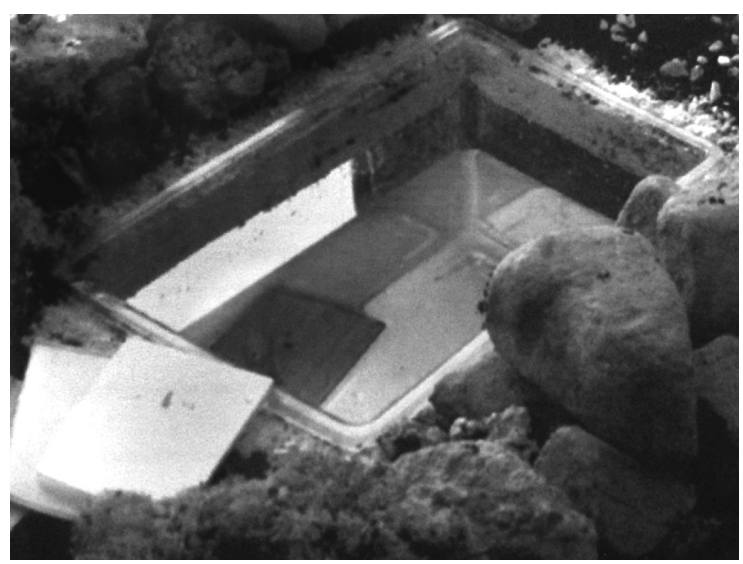

Source: élaboration propre

Le récipient oblong d'un aquarium a fait figure de lac. Pour la construction du sol, on a utilisé du papier pour maquettes, du sable, des cailloux et de la terre; de même pour les alentours. On a construit les barges avec du liège, du bois, de verre et de l'aluminium, toutes coupées par couples, une grande et une petite. Un petit lion en plastique figurait Beny et 10 boîtes d'allumettes drapées de papier blanc marqué d'une croix rouge et de l'inscription "fragile" représentaient les étudiants. Les boîtes étaient aussi enveloppées d'une membrane imperméable pour que les "médicaments" soient protégés de l'eau. Enfin, des feuilles A4 et des crayons étaient tenus à la disposition des enfants.

\section{Procédure}

La procédure expérimentale a suivi quatre phases distinctes:

Première phase. On demande aux enfants de nommer un à un les objets et le matériel de l'expérience. S'ils éprouvent des difficultés à reconnaître certains objets, on les nomme en leur expliquant de quoi il s'agit et on n'arrête la discussion avec eux que lorsqu'on est certain qu'ils sont en position d'utiliser les mêmes mots - et pour les mêmes objets-que les chercheurs. Par la suite, on explique aux enfants que l'on va jouer à un jeu - trouver les choses qui flottent - avec ces objets. Puis, on leur raconte une histoire courte. Aux enfants des groupes $A$ et $B$ on raconte I'histoire de Beny, fils du "roi des lions" qui, pourchassé par son méchant frère, arrive devant un lac. Ne sachant pas nager, il doit repérer lesquelles des barges qu'il y trouve peuvent lui permettre de traverser le lac. Aux enfants du groupe C on raconte I'histoire d'une mission humanitaire d'étudiants en médecine ayant pour but de transporter des médicaments en Irak. Durant leur marche, ils se trouvent devant un lac qu'ils doivent eux aussi traverser après avoir fait le bon choix de barges. Le problème est donc le même; ce qui change est I'histoire.

Deuxième phase. On demande aux enfants de prédire quelles barges vont flotter et lesquelles vont chavirer et pour quelle raison. Le but est d'examiner leur critère de choix des barges, ainsi que leurs explications. La question par laquelle commence la discussion est: "Quelles barges penses-tu que Beny (ou les étudiants) peut utiliser pour ne pas couler et pourquoi?"

Troisième phase. Une fois les prédictions et les explications des enfants données, on leur demande de faire des essais avec les barges pour vérifier leurs hypothèses. Ils sont libres de faire autant d'essais qu'ils le veulent. S'ils éprouvent des difficultés à commencer, on les encourage.

Quatrième phase. Enfin, on demande aux enfants de produire des instructions pour le jeu, de manière que les autres enfants puissent, en lisant ces instructions, faire traverser le lac à Beny/ aux étudiants. Les enfants sont libres de prendre des notes comme ils le veulent. Lorsqu'ils ont terminé, on leur demande éventuellement des éclaircissements.

\section{Questions de recherche}

On a interrogé les productions écrites des enfants de deux façons:

- La première question est liée au genre et aux caractéristiques de la production écrite des enfants quand ils essayent de construire un texte particulier. Ce texte doit, d'un côté, contenir les éléments qualitatifs 
fondamentaux pour la solution d'un problème élémentaire de physique et, de l'autre, permettre aux autres enfants de comprendre cette solution.

- La deuxième question est relative à l'évolution des productions écrites en fonction de l'âge des enfants.

Cadre d'analyse de productions écrites des enfants

Les productions écrites des enfants ont été traitées à deux niveaux:

- Premièrement, on a procédé à une description des productions et

- Deuxièmement, on les a évaluées sur deux dimensions: leur exhaustivité et leur fonctionnalité.

Ont été considérées comme exhaustives les notations comprenant tous les éléments nécessaires à la traversée du lac, à savoir:

- quelles sont les barges qui flottent et celles qui coulent,

- une incitation de choix et d'usage de celles qui flottent et

- des références aux éléments de base de I'histoire et, surtout, du problème à résoudre.

Ont été considérées comme fonctionnelles les notations dont l'organisation et le flux d'informations étaient susceptibles de faciliter la compréhension des autres enfants et, par conséquent, de leur permettre de satisfaire les exigences du jeu.

\section{Résultats}

Nous avons traité les réponses recueillies au cours des entretiens selon quatre niveaux. À l'intérieur de chaque niveau nous avons distingué différentes catégories d'écrits :

\section{Premier niveau: les caractéristiques} descriptives des notations

Les écrits des enfants ont été répartis selon cinq catégories en fonction de leurs caractéristiques descriptives.

- Première catégorie: productions écrites ayant la forme d'un texte écrit, certaines sous forme de liste d'instructions, d'autres sous forme linéaire (succession des mots ou des phrases à la même ligne) (Figure 1).

- Deuxième catégorie: écrits contenant à la fois du texte et du dessin (Figure 2).
- Troisième catégorie: notes ne comportant que des dessins (Figure 3).

- Quatrième catégorie: dessin se limitant au matériel utilisé (Figure 4).

- Cinquième catégorie: certains enfants, malgré les encouragements des chercheurs, n'arrivent à créer aucun type d'instructions écrites.

Figure 1

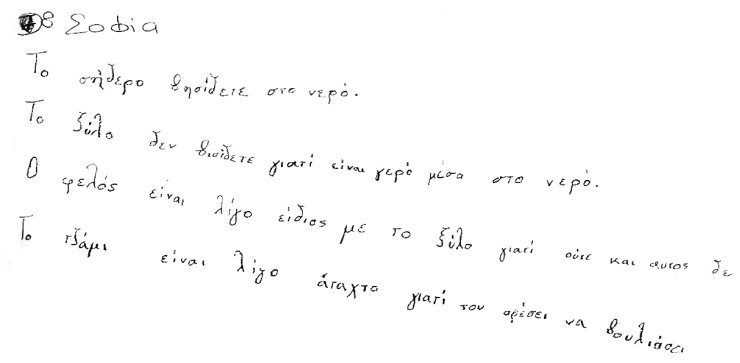

Figure 2

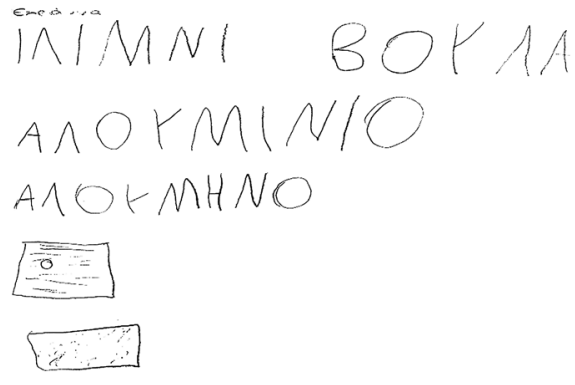

Figure 3
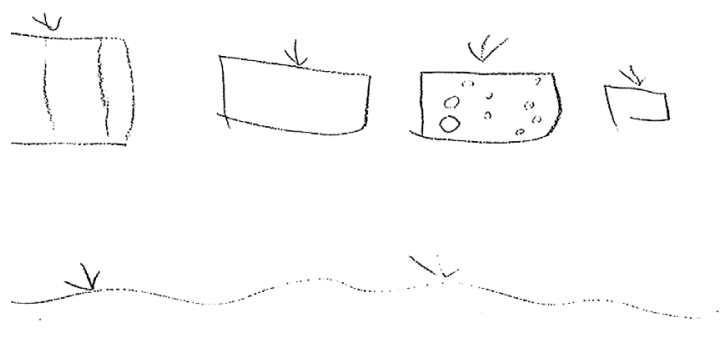

Figure 4

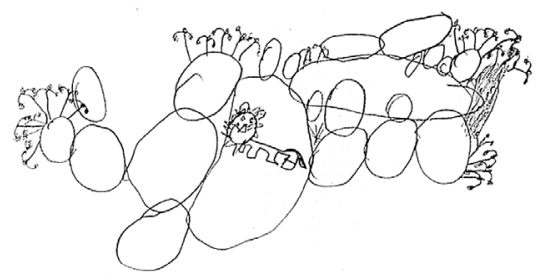

Source: élaboration propre 
Le Tableau 1 présente les fréquences des différents genres de productions écrites des enfants des trois groupes.

Tableau 1.

Fréquences des différents genres de productions

écrites des enfants des trois groupes

\begin{tabular}{|c|c|c|c|c|c|c|}
\hline & \multicolumn{2}{|c|}{ Ecriture } & \multirow{2}{*}{$\begin{array}{l}\text { Dessin et } \\
\text { écriture }\end{array}$} & \multirow{2}{*}{ Dessin } & \multirow{2}{*}{$\begin{array}{l}\text { Dessin du } \\
\text { matériel }\end{array}$} & \multirow{2}{*}{$\begin{array}{c}\text { Pas de } \\
\text { notation }\end{array}$} \\
\hline & Texte & Liste & & & & \\
\hline \multirow{2}{*}{$\begin{array}{l}\text { Groupe } A \\
(5-6 \text { ans) } \\
N=28\end{array}$} & \multicolumn{2}{|c|}{$5(17,9 \%)$} & \multirow{2}{*}{$1(3,6 \%)$} & \multirow{2}{*}{$18(62,3 \%)$} & \multirow{2}{*}{$2(7,1 \%)$} & \multirow{2}{*}{$2(7,1 \%)$} \\
\hline & 3 & 2 & & & & \\
\hline \multirow{2}{*}{$\begin{array}{l}\text { Groupe B } \\
(7-8 \text { ans) } \\
N=31\end{array}$} & \multicolumn{2}{|c|}{$17(54,8 \%)$} & \multirow{2}{*}{$6(19,4 \%)$} & \multirow{2}{*}{$6(19,4 \%)$} & \multirow{2}{*}{-} & \multirow{2}{*}{$2(6,4 \%)$} \\
\hline & 10 & 7 & & & & \\
\hline \multirow{2}{*}{$\begin{array}{l}\text { Groupe C } \\
\text { (II-I2 ans) } \\
N=32\end{array}$} & \multicolumn{2}{|c|}{$31(96,9 \%)$} & \multirow{2}{*}{-} & \multirow{2}{*}{$1(3,1 \%)$} & \multirow{2}{*}{-} & \multirow{2}{*}{-} \\
\hline & 26 & 5 & & & & \\
\hline \multirow[b]{2}{*}{ Total } & & & \multirow{2}{*}{7} & \multirow{2}{*}{25} & \multirow{2}{*}{2} & \multirow{2}{*}{4} \\
\hline & 39 & 14 & & & & \\
\hline
\end{tabular}

Source: élaboration propre

On observe un passage du groupe $A$, où les dessins dominent, au groupe $C$, où l'écriture prend le pas. Les productions du groupe $B$ présentent un intérêt particulier, étant donné qu'elles montrent d'une manière plus claire la transition du dessin à l'écriture.

\section{Deuxième niveau: exhaustivité et fonctionnalité}

Les écrits des enfants ont été classés en fonction de leur exhaustivité en trois grandes catégories: ceux qui sont exhaustifs, ceux qui ne le sont que partiellement et enfin ceux qui ne le sont pas du tout. Le critère est évalué comme suit:

- Exhaustivité: Les écrits présentent l'ensemble des éléments déjà mentionnés (choix des barges qui flottent, critères de choix et référence à I'histoire).

- Exhaustivité partielle: Les écrits ne présentent qu'un ou deux des trois éléments.

- Absence d'exhaustivité: Aucun élément permettant aux autres enfants de réussir l'activité n'est présent.

Le Tableau 2 présente les fréquences des écrits des enfants des trois groupes du point de vue de leur exhaustivité. 
Tableau 2.

Fréquences des écrits des enfants des trois groupes

du point de vue de leur exhaustivité

\begin{tabular}{l|c|c|c}
\hline \multicolumn{2}{c}{ Exhaustivité } & $\begin{array}{c}\text { Exhaustivité } \\
\text { partielle }\end{array}$ & $\begin{array}{c}\text { Absence } \\
\text { d'exhaustivité }\end{array}$ \\
\hline $\begin{array}{l}\text { Groupe A } \\
(\mathbf{5}-\mathbf{6} \text { ans) } \\
\mathbf{N}=\mathbf{2 8}\end{array}$ & $3(10,8 \%)$ & $2(7,2 \%)$ & $21(75 \%)$ \\
\hline $\begin{array}{l}\text { Groupe B } \\
(\mathbf{7 - 8} \text { ans) } \\
\mathbf{N}=\mathbf{3 I}\end{array}$ & $1(3,2 \%)$ & $14(45,2 \%)$ & $14(45,2 \%)$ \\
\hline $\begin{array}{l}\text { Groupe C } \\
(\mathbf{I I - I 2} \text { ans) } \\
\mathbf{N}=\mathbf{3 2}\end{array}$ & $8(25 \%)$ & $23(71,9 \%)$ & $1(3,1 \%)$ \\
\hline \begin{tabular}{l} 
Total \\
\hline \hline
\end{tabular} & 12 & 39 & 36 \\
\hline
\end{tabular}

Source: élaboration propre

On observe que les productions des enfants s'améliorent avec l'âge sur le plan de l'exhaustivité. Peu d'enfants $(10,8 \%$ du groupe A et 3,2\% du groupe B), même parmi les plus âgés (25\%), aboutissent à des productions tout à fait satisfaisantes. Toutefois, les résultats du groupe $B$ et plus encore du groupe $C$ montrent une amélioration systématique.

Concernant la fonctionnalité, les écrits des enfants se divisent en deux catégories: ceux dont l'étude permet la compréhension des éléments de base pour traverser le lac sont considérés comme satisfaisants et ceux qui ne rendent pas possible cette compréhension.

Le tableau 3 présente les fréquences des écrits des enfants des trois groupes par rapport à leur fonctionnalité.

Tableau 3.

Fréquences des écrits des enfants des trois groupes par rapport à leur fonctionnalité

\begin{tabular}{l|c|c}
\hline \multicolumn{2}{c}{ Fonctionnels } & Non fonctionnels \\
\hline $\begin{array}{l}\text { Groupe A } \\
\text { (5-6 ans) } \\
\mathbf{N}=\mathbf{2 8}\end{array}$ & - & $26(92,9 \%)$ \\
\hline $\begin{array}{l}\text { Groupe B } \\
\text { (7-8 ans) } \\
\mathbf{N}=31\end{array}$ & $10(32,3 \%)$ & $19(61,29 \%)$ \\
\hline $\begin{array}{l}\text { Groupe C } \\
\text { (11-12 ans) }\end{array}$ & $22(68,75 \%)$ & $10(31,25 \%)$ \\
$\mathbf{N}=\mathbf{3 2}$ & & \\
\hline Total & 32 & 55 \\
\hline \hline
\end{tabular}

Source: élaboration propre 
Comme on peut le constater dans le tableau 3, la fonctionnalité des productions des enfants suit I'âge: aucun enfant du groupe $A$ ne produit de notation satisfaisante, alors que 1 enfant sur 3 du groupe B et 2 sur 3 du groupe $C$ y parviennent.

\section{Discussion}

Dans la recherche présentée ici on a étudié l'évolution des caractéristiques et de I'utilisation des prises de notes dans le cadre d'une activité scientifique chez des enfants de 5 à 12 ans. Le premier résultat digne d'intérêt que présente cette étude est la faculté des enfants à comprendre l'intérêt des notes "pour se rappeler" et "pour pouvoir dire après à leurs camarades ce qu'ils ont fait", au point d'en créer qui satisfassent ce but. Même les enfants d'âge préscolaire répondent à ce problème de manière satisfaisante, étant donné qu'ils comprennent l'enjeu et ils prennent des notes.

Les formes de notations observées sont les suivantes: (1) Texte avec ou sans liste, (2) Dessin et écrit, (3) Dessin de la procédure et (4) Dessin du matériel. Toutefois, les productions des enfants diffèrent, tant au niveau quantitatif qu'au niveau qualitatif. Sur le plan quantitatif, on observe une augmentation presque linéaire du degré d'exhaustivité et du degré de fonctionnalité des notations par rapport à l'âge des enfants. Ces résultats semblent confirmer des résultats analogues, produits dans le cadre d'une recherche antérieure concernant des enfants d'âge préscolaire (Tantaros, Koliopoulos, Papandreou \& Ravanis, 2003). Bien sûr, une estimation systématique de l'exhaustivité et de la fonctionnalité des notations des enfants pourrait être faite non par les chercheurs, comme c'est le cas dans cette étude, mais par d'autres agents (éducateurs, camarades, parents, etc.). Cette autre piste de recherche pourrait nous offrir des renseignements supplémentaires sur leurs caractéristiques comme la stabilité de l'utilisation ou les champs d'application de ces notes.

Le deuxième résultat significatif, sur le plan qualitatif cette fois, est lié à l'évolution morphologique des notations des enfants. La caractéristique principale de cette évolution est l'augmentation de l'écrit et la diminution concomitante du dessin. Seuls les plus petits utilisent le dessin comme forme symbolique préférentielle de notation et cela paraît s'expliquer par la faiblesse de leurs compétences à l'écrit. Cela dit, si les plus grands préfèrent s'exprimer par écrit, ils évitent d'utiliser d'autres formes symboliques de notation plus "scientifiques", comme la liste. En effet, en grandissant, les enfants produisent moins de notations du type "liste d'actions" et recourent davantage à des productions du type "texte continu". La succession des notations de type "dessin" - "liste" - "texte" pourrait marquer les trois manières dominantes successives de s'exprimer par écrit aux trois niveaux scolaires correspondant à l'âge des enfants de chaque groupe. II serait intéressant d'examiner les raisons pour lesquelles les enfants abandonnent progressivement les représentations graphiques, telles que la classification des données dans le cadre d'une activité expérimentale, alors qu'en sciences physiques elles sont considérées comme des formes de notation fonctionnelles pour exposer et communiquer les connaissances spécialisées. Peut-être les enfants utilisent les moyens d'apprentissage dominant dans les pratiques des enseignants (Mackenzie, 2011, 2014).

Cette question entre aussi en relation avec l'axe de recherche portant sur les représentations des enfants concernant les notions de "submersion" et de "flottaison" des objets. II serait aussi intéressant de voir si —et 
comment- ces représentations influencent les systèmes de notations des enfants. Une telle investigation est en cours.

Plus généralement, le rôle des pratiques notationnelles dans l'appropriation des sciences physiques n'est toujours pas pleinement établi ; certains résultats, extrêmement limités, permettent de dire qu'elles peuvent faciliter la compréhension des concepts scientifiques par les élèves, mais rien n'est établi quant à un rapport causal entre notations et apprentissage (Schneeberger \& Vérin, 2009). À l'école, les écrits de travail tels que notes ou schémas provisoires sont rares et marginaux (Vérin, 1995). Pour autant, leur production permet la mobilisation de la pensée des élèves et ils peuvent servir de support à l'approfondissement du travail d'initiation scientifique à l'école. II semble donc que les résultats cette recherche renvoient, au plan didactique et éducatif, à l'investissement temporel nécessaire aux pratiques notationnelles en classe de sciences physiques et expérimentales. Ces résultats soulignent l'importance de l'enrichissement des moyens par lesquelles les prises de note aident les élèves à assimiler des concepts scientifiques.

Mais bien entendu, comme il ne s'agit que d'une étude de cas purement qualitative avec un échantillon limité, il convient d'être prudent. II est évident que la valeur éducative de l'activité effectuée, doit être confirmée non seulement dans des conditions expérimentales d'enseignement, mais aussi dans des recherches quantitatives et surtout dans des conditions "normales" de classe scolaire.

\section{Sur les auteurs}

Spyridon Tantaros est Professeur Associé de Psychologie du Développement, Université d'Athènes. II a fait des études de Psychologie à l'Université de Montpellier III (Diplôme de base) et de Toulouse Le Mirail (Master et Doctorat).

Konstantinos Ravanis est Professeur en Didactique de la Physique, Université de Patras. II a fait des études en Physique et en Sciences de l'Éducation (maîtrises), Didactique de la Physique à I'Université Paris VII (Master) et Didactique de la Physique (Doctorat à l'Université de Patras).

Dimitris Koliopoulos est Professeur en Didactique des Sciences Physiques et en Muséologie Scientifique, Université de Patras. II a fait des études en Physique (Maîtrise à l'Université de Thessaloniki), de Didactique de la Physique à l'Université Paris VII (Master) et Didactique de la Physique (Doctorat à I’Université de Patras).

Kalypso Sarigianni est institutrice à l'école maternelle en Grèce, elle a fait sa Maîtrise en Sciences de l'Éducation à l'Université de Patras.

Evmorfia Sotiropoulou est institutrice à l'école maternelle en Grèce, elle a fait sa Maîtrise en Sciences de l'Éducation à l'Université de Patras.

\section{Références}

Bovair, S. \& Kieras, D. E. (1991). Toward a Model of Acquiring Procedures from Text. In Rebecca Barr, Michael L. Kamil, Peter B. Mosenthal \& P. David Pearson (eds.). Handbook of Reading Research, Volume II, 206-229. New York \& London: Longman.

Canedo-Ibarra, S. P.; Castelló-Escandell, J.; García-Wehrle, P. \& MoralesBlake, A. R. (2010). Precursor Models Construction at Preschool Education: An Approach to Improve Scientific Education in the Classroom. Review of Science, Mathematics \& ICT Education, 4 (1), 
41-76. Disponible à: http://resmicte.lis.upatras.gr/index.php/review/ article/view/134/264

Catel, L. (2001). Écrire pour apprendre? Écrire pour comprendre? État de la question. Aster, 33, 17-47. Disponible à: http://ife.ens-lyon.fr/ publications/edition-electronique/aster/ASTER_2001_33 17.pdf

Cros, P. \& Respaud, S. (2001). Articulation entre des pratiques d'écriture et la construction des savoirs à l'école primaire: une étude de cas. Aster, 33, 163-188. Disponible à: http://ife.ens-lyon.fr/publications/editionelectronique/aster/ASTER_2001_33 163.pdf

Dixon, P.; Faries, J. \& Gabrys, G. (1998). The Role of Explicit Actions-Statements in Understanding and Using Written Directions. Journal of Memory and Language, 27 (6), 649-667.

Dumas-Carré, A.; Weil-Barais, A.; Ravanis, K. \& Shourcheh, F. (2003). Interactions maître-élèves en cours d'activités scientifiques à l'école maternelle: approche comparative. Bulletin de Psychologie, 56(4), 493-508. Disponible à: https://www.academia.edu/542708/Dumas_ Carr\%C3\%A9_A._Weil-Barais_A._Ravanis_K._Shourcheh_F._2003_. Interactions ma\%C3\%AEtre-\%C3\%A91\%C3\%A8ves en cours_d_activit\%C3\%A9s_scientifiques_\%C3\%A0_I_\%C $\overline{3}$ $\%$ A 9 cole_maternelle_approche_comparative._Bulletin_de Psychologie_56_4_493-508

Ferreiro, E. \& Gómez-Palacio, M. (1988). Lire-écrire à l'école: comment s'y apprennent-ils?: analyse des perturbations dans les processus d'apprentissage de la lecture et de l'écriture: recherche conduite au Mexique. Lyon: Centre régional de documentation pédagogique, CRDP.

Fillon, P. \& Vérin, A. (2001). Écrire pour comprendre les sciences. Aster, 33, 3-16. Disponible à: http://ife.ens-lyon.fr/publications/edition-electronique/aster/ASTER_2001_33_3.pdf

Fragkiadaki, G. \& Ravanis, K. (2015). Preschool Children's Mental Representations of Clouds. Journal of Baltic Science Education, 14 (2), 267-274. Disponible à: https://www.academia.edu/12302143/ Fragkiadaki_G._and_Ravanis_K._2015_._Preschool_children_s_ mental_representations_of_clouds._Journal_of_Baltic_Science_ Education_14_2_267-274

Ganier, F. (2002). L'analyse des fonctionnements cognitifs: un support à I'amélioration de la conception des documents procéduraux. Psychologie Française, 47 (1), 53-64.

Ganier, F.; Gombert, J.-E. \& Fayol, M. (1998). Élaborer des représentations pour l'action: le traitement d'instructions à partir de textes et d'illustrations. Revue de Psychologie de /'Éducation, 3, 141-169.

Garcia-Milà, M.; Teberosky, A. \& Martí, E. (2000). Anotar para resolver una tarea de localización y memoria. Infancia y Aprendizaje: Journal for the Study of Education and Development, 90, 51-70. Disponible à: http://www.researchgate.net/publication/233493844_Anotar para_resolver_una_tarea_de_localizacin_y_memoria_Notetaking_ to_solve_a_task_of_location_and_memory

Kohn, A. S. (1993). Preschoolers' Reasoning about Density: Will It Float? Child Development, 64, 1637-1650.

Koliopoulos, D.; Tantaros, S.; Papandreou, M. \& Ravanis, K. (2004). Preschool Children's Ideas about Floating: A Qualitative Approach. Journal of Science Education, 5 (1), 21-24. Disponible à: https://www.academia. edu/273045/Koliopoulos_D._Tantaros_S._Papandreou_M. Ravanis_K._2004_._Preschool_Childrens_Ideas_About_Floating_a_ Qualitative_Approach._Journal_of_Science_Education_5_1_21-24 
Lemeignan, G. \& Weil-Barais, A. (1993). Construire des concepts en Physique. Paris: Hachette.

Mackenzie, N. M. (2011). From Drawing to Writing: What Happens when You Shift Teaching Priorities in the First Six Months of School? Australian Journal of Language \& Literacy, 34 (3), 322-340. Disponible à: https://www.academia.edu/1105001/Mackenzie_N._M._2011_._ From_drawing_to_writing_What_happens_when_you_shift_ teaching_priorities_in_the_first_six_months_of_school_Australian_ Journal_of_Language and_Literacy_34_3

Mackenzie, N. M. (2014). Transitions to School and Emergent Writers. In Bob Perry, Sue Dockett \& Anne Petriwskyj (eds.). Transitions to School: International Research, Policy and Practice, 89-102. London: Springer. Disponible à: https://www.academia.edu/5873484/ Mackenzie_N.M._2014_._Transitions_to_school_and_emergent_ writers._In_B._Perry_S._Dockett_and_A._Petriwskyj_Eds. Transitions_to_school_International_research_policy_and_practice_ pp._89-102_Springer

Martí, E. (1997). Les débuts de la capacité notationnelle: implications didactiques. Skhôle: Cahier de la Recherche et du Développement, 7, 219-237.

Martí, E. \& Pozo, J. I. (2000). Más allá de las representaciones mentales: la adquisición de los sistemas externos de representación. Infancia y Aprendizaje, 90, 11-30. Disponible à: http://www.researchgate. net/publication/39138087_Mas_all_de_las_representaciones mentales_la_adquisicin_de_los_sistemas_externos_de_ representacin

Piaget, J. (1930/1974). The Child's Conception of Physical Causality. London: Transaction Publishers.

Piaget, J. \& Inhelder, B. (1942/1974). The Child's Construction of Quantities. London: Routledge \& Kegan Paul.

Pramling, N. \& Pramling-Samuelsson, I. (2001). "It is Floating 'Cause there is a Hole'": a Young Child's Experience of Natural Science. Early Years: An International Research Journal, 21 (2), 139-149.

Ravanis, K. (2011). L'écrit et l'activité notationnelle dans l'enseignement de la physique: questions actuelles pour la recherche et les pratiques des enseignants. Journal of Didactics, 2 (2), 157-169. Disponible à: https:// www.academia.edu/2499364/Ravanis_K._2011_._L_\%C3\%A9crit_ et_I_activit\%C3\%A9_notationnelle_\%C3\%A0_I_enseignement_ de_la_physique_questions_actuelles_pour_la_recherche_et_les_ pratiques_des_enseignants._Journal_of_Didactics_2_2_157-169

Ravanis, K.; Christidou, V. \& Hatzinikita, V. (2013). Enhancing Conceptual Change in Preschool Children's Representations of Light: A SocioCognitive Approach. Research in Science Education, 43 (6), 2257-2276. Disponible à: https://www.academia.edu/5606612/Enhancing_ conceptual_change_in_preschool_children_s_representations_of_ light_a_socio-cognitive_approach

Ravanis, K.; Papamichaël, Y. \& Koulaidis, V. (2002). Social Marking and Conceptual Change: the Conception of Light for Ten-Year Old Children. Journal of Science Education, 3 (1), 15-18. Disponible à: https:// www.academia.edu/273046/Ravanis_K._Papamicha\%C3\%ABI_Y._ Koulaidis_V._2002_._Social_marking_and_conceptual_change_ The_conception_of_light_for_ten-year_old_children._Journal_of_ Science_Education_3_1_15-18

Schneeberger, P. \& Vérin, A. (2009). Développer des pratiques d'oral et $d$ 'écrit en sciences: Quels enjeux pour les apprentissages à l'école? 
Lyon: Institut national de recherche pédagogique, INRP. Disponible à: http://ife.ens-lyon.fr/editions/editions-electroniques/developperdes-pratiques-doral-et-decrit-en-sciences-cahier-des-illustrationsschemas-et-figures

Tantaros, S.; Koliopoulos, D.; Papandreou, M. \& Ravanis, K. (2003). Notational Practices within Preschoolers' Constructions of Scientific Knowledge. Paper Presented at the 10 $10^{\text {th }}$ EARLI Biennial Conference, European Association for Research on Learning and Instruction, EARLI, University of Padova, Padova, Italy, 26-30 August 2003.

Vérin, A (1995). Mettre par écrit ses idées pour les faire évoluer en sciences. Repères, 12, 21-36.

Weil-Barais, A. \& Bouda, N. (2004). Contexte social et interactionnel d'activités expérimentales à l'école primaire. Aster, 38, 211-236. Disponible à: http://documents.irevues.inist.fr/bitstream/handle/2042/8837/ ASTER_2004_38_211.pdf?sequence $=1$

Yin, K. R. (2014). Case Study Research. Design and Methods. London: Sage Publications. 